\title{
Entornos virtuales de aprendizaje complejos e innovadores: Una experiencia de creación participativa desde el paradigma emergente ${ }^{1}$
}

\author{
Complex and Innovative Virtual Learning Environments: An Experience of Participatory \\ Creation from an Emergent Paradigm ${ }^{2}$
}

\section{Ambientes virtuais de aprendizagem complexos e inovadores: uma experiência de criação participativa, partindo do paradigma emergente ${ }^{3}$}

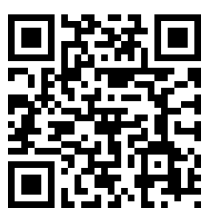

María Florencia Morado ${ }^{4}$ Universidad Nacional de Costa Rica Heredia, Costa Rica morado.florencia@gmail.com http://orcid.org/0000-0002-6588-4981

Recibido • Received • Recebido: 21 / 09 / 2016

Corregido • Revised • Revisado: 28 / 11 / 2017

Aceptado • Accepted • Aprovado: 15/ 12 / 2017

\begin{abstract}
Resumen: Este artículo surge de un proceso de investigación acción participativa que tuvo como objetivo analizar los lineamientos del paradigma emergente y proponer una alternativa para la construcción de entornos virtuales de aprendizaje (EVA) epistemológicamente coherente con dicho paradigma. La construcción de entornos virtuales de aprendizaje se presenta como un desafío para el personal docente, especialmente para quienes, epistemológicamente, se adhieren a las concepciones del paradigma emergente, que encuentra que las herramientas disponibles cercenan sus capacidades pedagógicas y creativas. Se realizaron dos talleres con un total de siete personas que completaron el proceso de investigación y construcción de sus entornos. En el proceso se definieron, en conjunto con el profesorado, qué lineamientos del paradigma emergente era necesario recuperar para la construcción de EVA. Para ello se exploraron herramientas tanto dentro de la plataforma Moodle como fuera de ella y se co-construyeron los entornos en un proceso recursivo de análisis, revisión y producción.
\end{abstract}

Palabras claves: Entornos virtuales de aprendizaje; paradigma emergente; investigación acción participativa.

${ }^{1}$ El artículo se origina a partir de la tesis de maestría: Comunidades de aprendientes en entornos virtuales: Una alternativa de educación sin distancias en el marco del paradigma emergente, presentada en la Universidad Nacional de Costa Rica en agosto de 2016.

${ }^{2}$ The article originates from the masters's degree thesis: Learners' communities in virtual environments: an educational alternative without distances within the framework of the emerging paradigm, presented at the Universidad Nacional , Costa Rica, in August, 2016.

${ }^{3}$ A origem desse artigo parte da seguinte tese de mestrado: Comunidades de estudantes em ambientes virtuais: uma educação alternativa, sem distâncias dentro do paradigma emergente, apresentada na Universidade Nacional da Costa Rica, em agosto de 2016.

${ }^{4}$ M.Sc. en Tecnología e Innovación en Educación por la UNA, Costa Rica. Licda. en Comunicación Social, UBA, Argentina. Diploma Superior en Ciencias Sociales con énfasis en Educación y Nuevas Tecnologías, FLACSO, Argentina. Actualmente coordinadora de la Maestría en Tecnología e Innovación Educativa de la Universidad Nacional de Costa Rica. Se desempeñó como profesora en la UBA, UTN, Universidad Maimónides, Argentina. 
doi: http://dx.doi.org/10.15359/ree.22-1.18

URL: http://www.una.ac.cr/educare

CORREO: educare@una.cr

\begin{abstract}
This paper starts with a participatory action research process, which aims to analyze the guidelines of the emerging paradigm and propose an alternative for building virtual learning environments (VLE) epistemologically consistent with this paradigm. Building virtual learning environments is presented as a challenge for teachers, especially for those who epistemologically adhere to the concepts of the emerging paradigm. Teachers find that the tools available encroach on their pedagogical and creative abilities. Two workshops were held with seven people who completed the research and the building process of their environments. In conjunction with the teaching staff, in the process were defined the guidelines of the emerging paradigm that were necessary to recover to build VLE; the teachers explored tools within the Moodle platform and others from Internet. Then, they co-constructed their environments in a recursive process of analysis and production.
\end{abstract}

Keywords: Virtual learning environments; emerging paradigm; participatory action research.

Resumo: Este artigo surge de um processo de pesquisa-ação participativa, que teve como objetivo analisar as diretrizes do paradigma emergente e propor uma alternativa para a construção de ambientes virtuais de aprendizagem (EVA em espanhol), epistemologicamente consistentes com este paradigma. A construção de ambientes virtuais de aprendizagem apresenta um desafio para os professores, especialmente aqueles que, epistemologicamente, assumem os conceitos do paradigma emergente, que consideram que as ferramentas disponíveis ajudam em suas habilidades pedagógicas e criativas. Foram realizadas duas oficinas com um total de sete pessoas que completaram o processo de pesquisa e construção de seus ambientes. No processo foram definidas, em conjunto com os professores, quais as diretrizes do paradigma emergente seriam necessárias para a construção de EVA. Para isso, foram exploradas ferramentas tanto da plataforma Moodle como outras, construindose ambientes, em um processo recursivo de análises, revisão e produção.

Palavras-chave: ambientes virtuais de aprendizagem, paradigma emergente, pesquisa-ação participativa.

\title{
Introducción
}

El desarrollo de este artículo es producto de un proceso de investigación acción participativa llevado adelante para la Tesis de la Maestría en Tecnología e Innovación en Educación en la Universidad Nacional de Costa Rica (Morado, 2016). En este proceso participó un grupo de docentes de la Universidad de la Salle de Costa Rica, entre julio de 2015 y febrero de 2016.

El origen de la demanda fue la necesidad de la Universidad de La Salle de crear una comunidad de práctica de docentes que aprendan, de forma participativa, a crear entornos virtuales de aprendizaje (EVA) con los lineamientos del paradigma emergente.

El proceso consistió en dos talleres de acompañamiento tecno-pedagógico con el personal docente donde se identificaron los lineamientos del paradigma emergente, el modelo de mediación pedagógica, las posibilidades y potencialidades de las tecnologías que ofrece la plataforma Moodle y la Web 2.0 y de qué manera se podían articular para la construcción los EVA de los cursos que desarrollaría el profesorado durante los siguientes cuatrimestres del calendario académico. 
La institución había tenido varios intentos de construcción de entornos virtuales de aprendizaje que no habían funcionado. En el momento de iniciar el proyecto se contaba con un uso fragmentado y de apoyo a la presencialidad de la herramienta para aulas virtuales Moodle. Sin embargo, el objetivo de las autoridades apuntaba a un proyecto integral de cursos bimodales y no como un repositorio de recursos y actividades.

Uno de los principales motivadores del proyecto fue constituir entornos virtuales que se basen en el concepto de "educación sin distancia" que propone Badilla (2015):

En el caso de que el aprendizaje se brinde en entornos virtuales, es necesario repensar lo que significa la corporalidad en la virtualidad por lo que la presencia, tanto de la persona docente como de todas las personas aprendientes, debe sentirse constantemente. (párr. 3)

Concretamente se indagó de qué manera deben construirse los entornos virtuales de aprendizaje para ir formando una nueva cultura, que reconozca los nuevos lenguajes, las nuevas reglas, nuevas formas de aprender en un mundo de sobreabundancia de información, donde el aprendizaje debe ser cada vez más flexible, autónomo y autorregulado. El proceso de investigación acción participativa constituyó una alternativa que se enfocó en crear EVA que recuperen la calidez del vínculo humano en una plataforma tecnológica.

Assmann (2002) asegura:

El ambiente pedagógico tiene que ser un lugar de fascinación e inventiva: no inhibir sino propiciar la dosis de ilusión común entusiasta requerida para que el proceso de aprender se produzca como mezcla de todos los sentidos. Transformación de los sentidos y significados, y potenciación de todos los sentidos con los que captamos corporalmente el mundo. Porque el aprendizaje es, antes que nada, un proceso corporal. (p. 28)

De esta manera, el desafío fue recuperar lo corporal, la presencia del profesorado y estudiantado en el entorno virtual, a través del diseño mismo del entorno y de las propuestas de interacción.

El llamado paradigma emergente, a diferencia del conocido como paradigma positivista, se caracteriza por tener una mirada compleja, integral y holística de los fenómenos.

Por ejemplo, Boff (2014) caracteriza el paradigma emergente de la siguiente forma:

- Totalidad / diversidad: todos los sistemas orgánicos e inorgánicos del universo están en constante adaptación y evolución a partir de interconexiones múltiples y diversas holísticamente interligadas. 
doi: http://dx.doi.org/10.15359/ree.22-1.18

URL: http://www.una.ac.cr/educare

CORREO: educare@una.cr

- Interdependencia / re-ligazón / autonomía relativa: todos los seres interligados se necesitan los unos a los otros para sobrevivir, pero cada uno goza de autonomía.

- Relación / campos de fuerza: todos los seres viven en un entretejido de relaciones; fuera de eso, no existen. Todo está dentro de campos de relaciones.

- Complejidad / interioridad: todo viene cargado de energías con diverso grado de complejidad e interacción y el universo puede ser visto como una totalidad inteligente y auto-organizada. Hay una organización interna que habilita la interacción; de esa forma, se crea un hilo conductor que provoca un caos generativo y un orden abierto a interacciones.

- Complementariedad, reciprocidad, caos: toda la realidad ocurre en la forma de partícula y onda, de energía y materia, orden y desorden, caos y cosmos y, a nivel humano, en forma de sapiens y de demens.

- Flecha del tiempo / entropía: todo lo que existe, preexiste y coexiste. Las relaciones en el tiempo tienen carácter irreversible; se abren a un futuro no predecible y no acabado, sino cargado de potencialidades.

- Destino común / personal: por la interconexión planetaria, todos los seres tenemos un destino común; dentro de él, cada ser se desarrolla individualmente.

- Bien cósmico / bien común particular: el bien común individual emerge a partir de la dinámica del bien general.

- Creatividad / destructividad: el ser humano interactúa permanentemente con su entorno, interviene en el ritmo de la naturaleza y, en su carácter de ser ético, puede evaluar las consecuencias de sus actos.

- Actitud holística / ecológica: la actitud de apertura y de interactividad permite superar el antropocentrismo histórico; se está en sinergia con el universo.

Siemens (2010) en el marco de dicho paradigma, asegura que el conocimiento es factible en la interacción no solamente entre personas, sino con el medio biológico, cultural y también entre sistemas informáticos. Afirma que "vivimos en una experiencia múltiple e integrada; es decir, vemos, conocemos y funcionamos de manera conectada. La vida, como el conocimiento, no es una actividad aislada, sino que es una parte rica e interconectada de lo que somos". (2010, p. 4). Por su parte, Downes (2005) explica cómo el conocimiento es posible en la interacción y desarrolla el concepto de emergencia de comportamientos complejos a partir de la interacción de unidades simples. La emergencia es la interpretación aplicada a las conexiones. 
En este sentido, las conversaciones permiten conectar e interactuar con el entorno, y es de especial relevancia en entornos virtuales, donde el cuerpo está ausente y el vínculo se da a partir de las interacciones conversadas. Maturana (2008) sostiene que"en el conversar construimos nuestra realidad con el otro. No es una cosa abstracta. El conversar es un modo particular de vivir juntos en coordinaciones del hacer y el emocionar. Por eso el conversar es constructor de realidades" ( $p$. 23). En entornos virtuales, las conversaciones constituyen la identidad y la presencialidad de las personas y es mediante la utilización de herramientas como foros, chats y correos electrónicos como se recuperará el cuerpo ausente y, a su vez, se acortarán lejanías afectivas, cognitivas, geográficas y emocionales (Badilla, 2015). La presencia del profesorado y el estudiantado debe ser evidente, la comunicación fluida y la retroalimentación oportuna. De esta manera se logrará propiciar un ambiente de aprendizaje activo y colaborativo para el estudiantado.

Ahora bien, ¿de qué manera se debe emprender esta transformación? Será a partir de la práctica significativa y no de capacitaciones teóricas vacías de contacto con la realidad, en un ida y vuelta de la práctica hacia la teoría, considerando qué reacciones y sensaciones producen en los aprendientes. Así se deberá encarar un proyecto, ya no de formación, más bien de transformación brindando la "oportunidad de aprender desde la práctica, desde lo concreto, desde la experiencia (como han hecho personas científicas y teóricas desde siempre)" (Badilla, 2015, párr. 9).

Este cambio de paradigma exige también cambios en quienes participan de los procesos educativos. Profesorado y estudiantado deberán desarrollar nuevas habilidades comunicacionales, de interacción con fuentes de información y construcción de información en diferentes formatos, para diferentes públicos y contextos (Cobo y Moravec, 2011).

El profesorado deberá ser capaz de preparar al estudiantado para lo inesperado, "Io nuevo brota sin cesar; nunca podemos predecir cómo se presentará... Y, una vez [que] sobrevenga ..., habrá que ser capaz de revisar nuestras teorías e ideas" (Morin, 1999, p. 14). De la misma manera, cambiará su rol, ya que no será quien posea todo el conocimiento, sino que deberá acompañar a personas de diferentes edades, contextos sociales, formación, conocimientos para crear una comunidad de aprendizaje donde el aprendizaje sea en múltiples vías.

La tecnología deberá formar parte del proceso en la educación del futuro; no deberá ser percibida como una herramienta aislada, sino que será un elemento constitutivo para investigar, conocer, comunicarse, cooperar, colaborar, y para simular entornos y escenarios. La tecnología es la que habilita la creación de espacios de socialización que fueron conceptualizados como comunidades virtuales por Rheingold (1996): se trata de "agregados sociales que surgen en la Red cuando una cantidad suficiente de gente lleva a cabo estas discusiones públicas durante un tiempo suficiente, con suficientes sentimientos humanos como para formar redes de relaciones personales en el espacio cibernético" (p. 20). En los espacios virtuales de aprendizaje, las conversaciones e interacciones tienen como objetivo la construcción de conocimiento. La tarea del profesorado es clave para incentivar, propiciar y orientar esas conversaciones. 
doi: http://dx.doi.org/10.15359/ree.22-1.18

URL: http://www.una.ac.cr/educare

CORREO: educare@una.cr

Wenger, White y Smith (2009) describieron las comunidades de práctica como aquellas comunidades que se conforman por personas con intereses similares y objetivos comunes y que requieren alta actividad de mediación para su éxito, pues "aprender juntos es un logro complejo que requiere un compromiso individual y comunal, aspiraciones e identidades" (Wenger et al., 2009, p. 58). En el proceso de esta investigación se conformó una comunidad de práctica conformada por quienes participaron del proceso, que, a la vez, analizaban los lineamientos del paradigma emergente, definían cuáles debían estar presentes en los entornos virtuales y aprendían a construir EVA tomando en cuenta estos lineamientos, en un espiral virtuoso de reflexión y acción.

Dos aspectos se tomaron en cuenta: (1) las características de las comunidades de aprendientes y su capacidad de adopción y asimilación de las TIC para la transformación de sus prácticas profesionales; y (2) cuáles herramientas son las más apropiadas para la construcción de entornos virtuales que respondan conceptualmente al paradigma emergente.

En cuanto al profesorado, se tuvo en cuenta el bagaje de competencias con que contaban al iniciar el proyecto, sobre todo las resistencias, temores e inseguridades. Se contemplaron las experiencias previas en su vínculo con la tecnología fuera del entorno educativo y de qué manera consideraban que la tecnología les permitía establecer entornos de aprendizaje colaborativos, flexibles y multisensoriales.

La tecnología puede incorporarse para responder a la lógica del paradigma positivista, de educación centrada en el personal docente y de clases expositivas, pero con nuevas herramientas. También tiene el potencial de crear nuevos entornos vitales. Se busca diferenciar el concepto de repositorio, que responde a un espacio donde se almacenan archivos, con el de la construcción de un entorno que favorece el vínculo y la mediación pedagógica. Las teorías recientes en los círculos educativos que tratan de llenar este bache, como el constructivismo y el aprendizaje activo, postulan que el alumnado crea conocimiento y significado activamente a través de la experimentación, la exploración y, la manipulación y prueba de las ideas en la realidad (Palloff y Pratt, 1999).

\section{Metodología}

A partir de un proceso de investigación acción participativa enmarcado en el paradigma interpretativo / constructivista, caracterizado por comprender las visiones de quienes participan en el proceso sobre la situación investigada (Mackenzie \& Knipe, 2006), se buscó analizar en conjunto con las personas participantes cuáles eran, desde su perspectiva, aquellos aspectos del paradigma emergente que debían estar presentes en la construcción de EVA. De acuerdo con Hernández, Fernández, Baptista (2010), la meta de la investigación es comprender la realidad teniendo en cuenta las percepciones y significados que se generan a partir de las experiencias de las propias personas participantes, en este caso, el profesorado. La investigación-acción fue 
una alternativa a las normas de la investigación descontextualizada; en lugar de centrarse en las encuestas y métodos estadísticos, el propósito de la investigación-acción era mejorar las formaciones sociales mediante la participación de los participantes en un proceso cíclico de la determinación de los hechos, la planificación, la acción de exploración y evaluación como se puede observar en la Figura 1 (Lewin 1948, citado en Somekh y Zeichner, 2009).

Fase 2

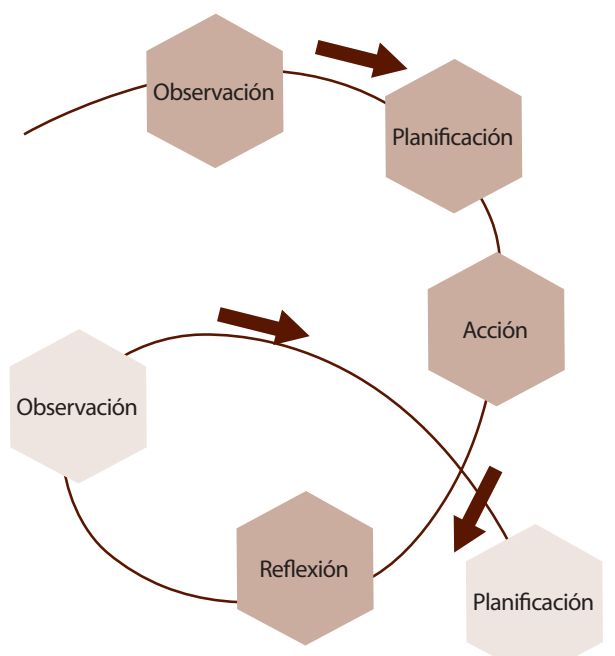

\section{Fase 1}

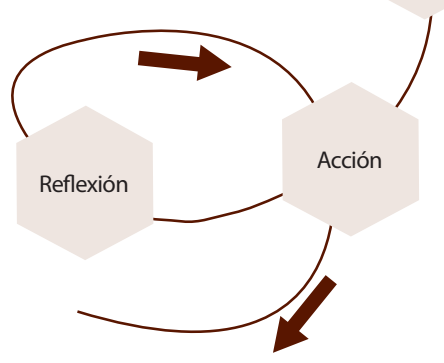

Figura 1: Modelo de investigación acción.

Nota: Elaboración propia.

El proceso de investigación acción participativa se hizo con siete docentes de la universidad que participaron de forma voluntaria del proceso completo, provenientes de las facultades de Administración, Educación, Psicología y Derecho. La muestra se definió por conveniencia vinculada con la participación de las personas en los Talleres de Acompañamiento TecnoPedagógico para la construcción de los EVA. 
doi: http://dx.doi.org/10.15359/ree.22-1.18

URL: http://www.una.ac.cr/educare

CORREO: educare@una.cr

El proceso completo consistió en:

Fase 0: Identificación de las necesidades indagando en los problemas actuales, urgencias a resolver, motivaciones, estado actual en relación con el conocimiento sobre EVA y recursos con los que se contaba.

Fase 1: Elaboración del diagnóstico que determinó que las necesidades eran de índole cognitiva, técnica e instrumental.

Fase 2: Diseño del proyecto a partir de plantear una serie de actividades en dos talleres de Acompañamiento-Tecno-Pedagógico que serían el espacio de aprendizaje y reflexión.

Fase 3: Desarrollo de los talleres en los que se construyeron los entornos virtuales y se definieron los lineamientos con los que se debían desarrollar.

Fase 4: Control operacional mediante acción- reflexión- acción donde se analizó si los entornos construidos respondían a los lineamientos definidos, se reflexionó sobre la experiencia y se hicieron propuestas de mejora.

Fase 5: Cierre del proyecto - Redacción de la tesis de maestría y socialización del documento final con las autoridades de la institución y con docentes que participaron.

Los instrumentos de recolección de datos fueron: a. Entrevistas focalizadas con guías de orientación al cuerpo docente involucrado voluntariamente. b. Consultas a informantes clave como lo fueron las autoridades de la institución. c. Cuestionarios de entrada y salida al profesorado antes de iniciar el proceso y al finalizar que indagaron sobre las necesidades, expectativas y recuperaron su relato de las experiencias vividas y significados asignados al proceso. d. Bitácora de las experiencias durante todo el proceso. Además, se generaron preguntas en los foros del entorno virtual de los talleres que permitieron obtener retroalimentación de las personas participantes. Se fueron redactando informes preliminares que se compartieron con el profesorado y que permitieron hacer ajustes en los talleres.

Si se considera que el producto final es una propuesta para la construcción de EVA y que la institución ha tenido intentos fallidos anteriores, fue fundamental, en esta oportunidad, involucrar a los actores en el diseño de la solución. Deesemodo, se buscó que fueran constructores activos en la definición del problema y en el análisis de las alternativas de solución y es a partir de un proceso de investigación acción participativa que se coconstruyó la solución en conjunto con las personas participantes involucradas. En tal proceso, como lo asevera Kemmis (2009), se cambiaron las prácticas de las personas, el decir sobre esas prácticas y las condiciones en las cuales las llevaban adelante.

Para el análisis de la información generada por los sujetos participantes se utilizaron los siguientes constructos (Morado 2016, p. 74). 
- ¿Cómo la presencialidad se construye en las conversaciones?

- ¿De qué manera el aprendizaje se genera en el hacer?

- ¿Cómo se evidencia que el conocimiento se genera en la interacción con otras personas y con entidades tecnológicas?

- ¿Cómo los entornos virtuales de aprendizaje están compuestos por servicios de gestión del aprendizaje (LMS) como punto de partida, pero se construyen a partir de las conexiones con otros espacios y aplicaciones fuera de ellos?

- ¿De qué forma los entornos virtuales se constituyen a partir de contenidos multimedia que permitan incentivar diferentes estilos cognitivos?

A la luz del análisis, se propusieron lineamientos para el desarrollo de EVA que respondieran al paradigma emergente y que guiaron su construcción. Una vez construidos los entornos con sus contenidos temáticos, recursos para el aprendizaje, propuestas de actividades, se los analizó en conjunto con las personas participantes a modo de coevaluación entre pares con el objetivo de que la mirada externa pueda validar que se habían construido, según los lineamientos que se crearon a lo largo del proceso.

\section{Resultados}

Se considera que la labor de enseñanza, que a efectos de este trabajo se debe entender como mediación, formación y acompañamiento al profesorado, deben constituir a su vez una tarea investigativa o una investigación en la acción (Carlino, 2010; Kemmis, 1993), por lo tanto, aquellas situaciones problemáticas y conflictivas se transforman en insumos para la construcción de soluciones coconstruidas con las personas docentes participantes que, a su vez, generan reflexión y aprendizaje sobre las propias prácticas profesionales. No se trata solo de alcanzar objetivos aplicando recetas preconfiguradas, sino más bien de comprender por qué algunas propuestas de capacitación no funcionaron en su momento o por qué algunas propuestas funcionaron en unos contextos y en otros contextos no. Se trata de ir analizando y creando propuestas suficientemente flexibles que puedan ajustarse a los diferentes contextos en que se trabaja. En tal sentido, la experiencia se documenta con el objetivo de dejar de ser una actividad privada de la persona formadora o del profesorado que participó del proceso, para convertirse en una "propiedad colectiva, sujeta a difusión, a crítica y al enriquecimiento por parte de la comunidad docente o de formadores de formadores" (Carlino, 2010, p. 123).

En las diferentes indagaciones, se hicieron presentes conceptos que provienen del paradigma emergente. Los de más relevancia para el profesorado fueron: aprendizaje, estudiantes, espacio, proceso, mediación, construcción e interacción. 
doi: http://dx.doi.org/10.15359/ree.22-1.18

URL: http://www.una.ac.cr/educare

CORREO: educare@una.cr

Desde la perspectiva del paradigma emergente estos conceptos significan:

- Aprendizaje: entendido como el proceso interactivo, multidimensional y complejo entre individuos y el entorno. El aprendizaje se da en múltiples dominios: físico, cognitivo, espiritual y emocional. A su vez, genera transformaciones en el saber sobre, saber hacer, saber dónde y saber ser (Siemens, 2010).

- Estudiantes: mejor definidos como aprendientes, es decir, individuos que están en capacidad de flexibilizarse, adaptarse, reestructurarse, interactuar, creary coevolucionar; seres que aprenden (Assman, 2002).

- Espacio: concebido como hábitat; es decir, un lugar de interacción que reúne las condiciones adecuadas para que la especie pueda residir y reproducirse, perpetuando su presencia.

- Proceso: es un conjunto de hechos o fases sucesivas de un fenómeno o de un hecho complejo; procesamiento o conjunto de operaciones a que se somete una cosa para elaborarla o transformarla.

- Mediación: mediación pedagógica entendida como la capacidad de sentir, intuir, vibrar emocionalmente; imaginar, inventar, crear y recrear; relacionar e interconectarse, autoorganizarse; informarse, comunicarse y expresarse; localizar, procesar y utilizar la inmensa información de la "aldea planetaria"; buscar causas y prever consecuencias; criticar, evaluar, sistematizar y tomar decisiones; pensar en totalidad (holísticamente).

- Construcción: construcción del conocimiento a partir de la interacción con elementos del entorno, conceptualización y aprendizaje en la creación de objetos concretos. Se produce entendimiento del mundo al crear artefactos, experimentar con ellos, para luego construir estructuras mentales que organicen y sinteticen la información y las vivencias de la vida cotidiana (Badilla y Chacón, 2004).

- Interacción: las interacciones son entre seres humanos entre sí y seres con objetos del entorno, tecnológico o no, con bases de datos y fuentes de información. También se conceptualiza como conectivismo. En los entornos virtuales la interacción entre seres humanos se concreta en las conversaciones; estas conversaciones constituyen la forma de estar presente en los entornos. La interacción permite la construcción del conocimiento puesto que depende del individuo, pero reside en el colectivo (Siemens, 2010).

El paradigma emergente asigna vital importancia al proceso de aprendizaje que se produce a partir de la mediación pedagógica entre estudiantes y docentes a raíz de la interacción. En tal proceso, el estudiantado es actor activo y autónomo en la construcción de los aprendizajes. La mediación pedagógica sucede en un espacio coconstruido entre aprendientes. 
Un entorno virtual, a diferencia de un repositorio de documentos, genera la posibilidad de construir un ambiente de aprendizaje donde las personas interactúan entre sí y con los recursos de aprendizaje, poniendo en juego sus emociones y conocimientos, construyendo conocimiento de manera activa y colaborativa junto con las otras personas estudiantes y docentes.

Los aspectos más importantes que se considerarán como principios de diseño para los ambientes de aprendizaje propuestos:

- Complejidad

- Multidimensionalidad

- Interactividad

- Interconexión

- Multiplicidad de conexiones

- Conversaciones como espacio para construir la realidad con otras personas

- Aprendizaje activo y colaborativo

- Práctica como espacio de consolidación de ideas

- Construccionismo

- Aprender haciendo

- Autoorganización

- Comunidades virtuales y de aprendientes

- Tecnología como espacio de socialización y como soporte para la construcción de comunidades

- Entornos de aprendizaje colaborativos, flexibles y multisensoriales

- Conectivismo entre seres humanos, información y tecnología

A continuación, se exponen las categorías desarrolladas en el marco de esta investigación y que permitieron el diseño de entornos virtuales con el paradigma emergente (Tabla 1): 
doi: http://dx.doi.org/10.15359/ree.22-1.18

URL: http://www.una.ac.cr/educare

CORREO: educare@una.cr

Tabla 1: Lineamientos para el desarrollo de EVA

\section{Orientación}

Todas las sesiones (presenciales y virtuales) están visibles en la plataforma.

Especifica fechas para cada una.

Determina cuáles son de presencia física y cuáles virtual.

Se accede fácilmente al programa del curso, los objetivos y calendario de actividades.

\section{Presencia virtual}

Claridad en la presentación de la sesión, introducción, desarrollo, recursos, lecturas y actividades.

Existe foro de consultas.

Las fechas de las semanas son correctas.

Además de la fecha, utiliza un título descriptivo de la sesión.

Desarrollo de contenidos

Introducción en pantalla principal.

Utiliza herramientas como etiquetas, libro y página para desarrollar la sesión.

Se utiliza un lenguaje cálido, ameno y comprensible.

Apela a los diversos estilos de aprendizaje mediante la utilización de texto, imágenes y videos.

Se etiquetan las diferentes instancias (Introducción, desarrollo, recursos, actividades)

\section{Diseño visual}

Utiliza una línea de diseño y colores uniformes en todo el curso.

\section{Recursos}

Los artículos o recursos para descargar en PDF se presentan con título y autor mediante el formato APA. Los recursos multimediales están embebidos dentro de la plataforma.

Los enlaces abren en página nueva.

Las referencias a páginas web están enlazadas.

\section{Actividades}

Utiliza las herramientas de las plataformas destinadas a la entrega de actividades (entrega de tareas, foros).

Especifica fechas de entrega.

Utiliza plataformas de web 2.0 externas para el desarrollo de actividades.

Promueve herramientas de trabajo colaborativo.

Nota: Morado (2016). 
Lograr la conformación de una comunidad de aprendientes requiere un rol activo del profesorado y propuestas de interacción orientadas a tal fin. Una delas actividades más importantes que debe llevar a cabo es, justamente, proponer un ambiente de interacción, de participación y de colaboración que evidencie la presencia de todo el estudiantado, así como la suya misma.

- Sus respuestas al estudiantado deben ser oportunas, con no más de 48 horas de demora.

- Cada vez que inicia una nueva semana, sesión o unidad de aprendizaje, según sea el caso, deberá anunciarlo a todo el estudiantado explicitando los objetivos de esa unidad, las actividades, fechas límite y, sobre todo, qué se espera que aprenda.

- Propiciará en foros participaciones activas y reflexivas, así como la interacción con las participaciones de las otras personas estudiantes.

- Deberá intervenir en las conversaciones orientando hacia los conceptos y contenidos de la sesión o alentando al estudiantado cuando van por el rumbo correcto.

- Cuando una persona estudiante no participe, indagará sobre el motivo de la no participación y le incentivará a expresar sus reflexiones. Si la no participación es por falta de comprensión del tema, profundizará sus explicaciones.

- Al finalizar el tiempo estipulado para las participaciones en foro deberá hacer una síntesis que recoja las participaciones del estudiantado. Esto permite, por un lado, dar cuenta de que el profesorado conoce todas las participaciones $y$, por otro, que puede construir una unidad de conocimiento a partir de las participaciones.

Palloff y Pratt (1999) definen la presencia en entornos virtuales como el grado en que una persona puede ser concebida como "real", pero la participación no únicamente se tiene que entender como que la persona estudiante publica un mensaje o envía un correo. Está relacionada con la calidad de la interacción y el sentido de pertenencia. Por lo tanto, el enfoque del ambiente de aprendizaje que se debe lograr apuntará a la colaboración, a alcanzar objetivos compartidos y al trabajo en equipos.

La presencia de estudiantes y docentes está en la participación. Las comunidades que se constituyen a partir de esta participación permitirán construir conocimiento de manera colaborativa. Las estrategias que promuevan la interacción facilitarán:

- Crear un clima de respeto y aceptación por la opinión de las otras personas

- Fortalecer capacidades argumentativas

- Sentir confianza hacia las otras personas 
doi: http://dx.doi.org/10.15359/ree.22-1.18

URL: http://www.una.ac.cr/educare

CORREO: educare@una.cr

- Lograr conexiones significativas entre sus participantes

- Crear compromiso no solo hacia el propio aprendizaje, sino hacia el aprendizaje de las demás personas

- Generar un nivel más profundo de comprensión

- Generar un sentido de pertenencia hacia el curso y hacia la institución

Wenger (2001) afirma que el compromiso facilita el proceso de aprendizaje al construir comunidades por medio de la inventiva, la energía social y el conocimiento emergente. Se generará una infraestructura de participación que incluya:

\section{Mutualidad}

1. Medios interactivos: espacios, tecnologías, medios para el encuentro

2. Tareas conjuntas: actividades para hacer de manera colaborativa

3. Periferia: encuentros fuera de los límites, encuentros casuales

\section{Competencia}

1. Iniciativa y experiencia: actividades que provocan la experiencia de compromiso, ocasiones para aplicar capacidades, idear soluciones y tomar decisiones; problemas que comprometen la energía, la creatividad y la inventiva

2. Responsabilidad: ocasiones para emitir juicios y hacer evaluaciones mutuas

3. Instrumentos: artefactos que apoyen la competencia, discursos, términos y conceptos

\section{Continuidad}

1. Memoria cosificadora: almacenes de información, documentación y rastreo; mecanismos de recuperación

2. Memoria participativa: encuentros entre generaciones, sistemas de aprendizaje, trayectorias paradigmáticas y narraciones (p. 283)

De tal forma que lo que guíe la construcción de los EVA sea no solo la presentación de contenidos de manera diversa sino la generación de espacios de interacción. 


\section{Conclusiones}

En la Universidad De La Salle el paradigma emergente se considera el paradigma orientador de las prácticas pedagógicas de la institución. Dicho paradigma atraviesa las prácticas académicas de forma transversal en el desarrollo de las clases presenciales. Sin embargo, en la construcción de entornos virtuales no estaban reflejados los lineamientos; sino que era un repositorio de documentos. No había evidencia de interacción humana, lo que contradice los lineamientos del paradigma emergente. En otros casos, no se trabajaba con entornos virtuales en absoluto. Esta situación se originaba principalmente por desconocimiento de cómo articular las herramientas tecnológicas con el paradigma emergente, lo que generaba frustración en el personal docente. Este reconocía, por un lado, la necesidad de incorporar tecnología, especialmente la bimodalidad en sus cursos; pero, por otro lado, no sabían cómo hacerlo para no contradecir sus convicciones epistemológicas para la práctica docente.

Se evidenció que es posible articular tecnología y paradigma emergente para la construcción de entornos virtuales. Las posibilidades de las herramientas están a disposición y el punto más relevante es cómo las tecnologías se articulan y configuran de forma tal que respondan a epistemologías novedosas. No es la herramienta misma, sino que las prácticas y configuraciones que se hacen con dicha herramienta les dan sentido. Para ello se hace necesario, primero, explorar, investigar, conocer diversas herramientas, y segundo, tener claridad en cuanto a los lineamientos del paradigma emergente, de modo que se puedan diseñar entornos y propuestas que estimulen diversos estilos de aprendizaje, que fomenten la colaboración entre pares, que estimulen los sentidos, y que propicien la producción de documentos complejos y multimedia.

La configuración de los entornos virtuales debe prestar especial atención a la orientación al estudiantado, a cómo se presentan los contenidos, a la inclusión de multimedios, cómo se presentan los recursos y cómo se diseñan las actividades, con el objetivo de constituir un ambiente de colaboración e interacción en el que emerja el aprendizaje a partir de la vinculación entre pares. Este ambiente de aprendizaje deberá garantizar ser un entorno sin distancias, donde la presencia virtual será tanto o más fuerte que la presencia física para la construcción del conocimiento. La creación del ambiente propicio será, entonces, un acto voluntario e intencional por parte del personal docente, quien se valdrá de las posibilidades tecnológicas disponibles.

El conjunto del entorno, así como la función docente y la interacción que propicia deberán lograr establecer un vínculo real y activo entre las personas participantes, estudiantes entre sí, y estudiantes con docentes. La presencia de docentes y estudiantes deberá ser evidente al utilizar los canales de comunicación, interacción y colaboración existentes, igual de fehaciente deberá ser la atención que se pone a la intervención de las otras personas. Se deberán brindar respuestas a partir de lecturas comprometidas con la participación de las demás personas. No se trata solamente de evidenciar la presencia individual y hasta egoísta, sino que la palabra de la otra persona debe ser tan importante como la propia, que será recuperada para la construcción del discurso y debe ser notoria y clara como el aporte de los demás individuos construye el propio aprendizaje. 
doi: http://dx.doi.org/10.15359/ree.22-1.18

URL: http://www.una.ac.cr/educare

CORREO: educare@una.cr

\section{Referencias}

Assmann, H. (2002). Placery ternura en la educación. Hacia una sociedad aprendiente. Madrid: Narcea.

Badilla, E. (06 de mayo de 2015). Educación sin distancia. La Nación. Recuperado de http://www. nacion.com/opinion/foros/eleonora-badilla-educacion-distancia 0 1485851420.html

Badilla, E. y Chacón, A. (2004). Construccionismo: Objetos para pensar, entidades públicas y micromundos. Revista Actualidades Investigativas en Educación, 4(1), 1-13. Recuperado de http://www.redalyc.org/articulo.oa?id=44740104

Boff, L. (2014). Características del nuevo paradigma emergente. Koinonia, 676. Recuperado de http://www.servicioskoinonia.org/boff/articulo.php?num=676

Carlino, P. (2010). Formación en servicio de profesores secundarios y universitarios para integrar la lectura y escritura en todas las materias. En H. M. Jacobo (Comp.), Complejidad, comunidades docentes y formación de profesores (pp. 121-142). Sinaloa: EDIUAS. Recuperado de https:// www.aacademica.org/paula.carlino/10.pdf

Carr, Wilfred y Kemmis, Stephen. (1986). Becomming critical. Education, knowledge and action research. London: Falmer.

Cobo, C. y Moravec, J. W. (2011). Aprendizaje invisible: Hacia una nueva ecología de la educación (Collecció Trasmedia XXI. Barcelona: Publicacions i Edicions de la Universitat de Barcelona. Recuperado de http://www.aprendizajeinvisible.com/download/Aprendizajelnvisible.pdf

Downes, S. (2005). Feature: E-learning 2.0. Elearn magazine, 10(1).

Hernández, R., Fernández, C., \& Baptista, P. (2010). Metodología de la investigación. México D.F.: Mcgraw-HILL / Interamericana Editores, S.A. de C.V.

Kemmis, S. (1993). Action research and social movement. A challenge for policy research. Education policy analysis archives, 1(1), 1-8. doi: https://doi.org/10.14507/epaa.v1n1.1993

Kemmis, S. (2009). Action research as a practice-based practice. Educational Action Research, 17(3), 463-474. doi : https://doi.org/10.1080/09650790903093284

Mackenzie, N., \& Knipe, S. (2006). Research dilemmas: Paradigms, methods and methodology. Issues in educational research, 16(2), 193-205.

Maturana, H. (2008). El sentido de lo humano. Buenos Aires: Juan Carlos Sáez.

Morado, F. (2016).Comunidades de aprendientes en entornos virtuales: Una alternativa deeducación sin distancias en el marco del paradigma emergente (Tesis de maestría). Universidad Nacional. San José, Costa Rica. 
doi: http://dx.doi.org/10.15359/ree.22-1.18

URL: http://www.una.ac.cr/educare

Morin, E. (1999). Los siete saberes necesarios para la educación del futuro. París: Unesco.

Palloff, R. M., y Pratt, K. (1999). Building learning communities in cyberspace. San Francisco: Jossey-Bass.

Rheingold, H. (1996). La comunidad virtual. Una sociedad sin fronteras. Barcelona: Gedisa.

Siemens, G. (2010). Conociendo el conocimiento. Canadá: Ediciones Nodos Ele. Recuperado de https://drive.google.com/file/d/OB7qpQvDV3vxvOFZBQVQxYTRPZFk/view

Somekh, B. y Zeichner, K. (2009). Action research for educational reform: Remodelling action research theories and practices in local contexts. Educational Action Research, 17(1), 5-21. doi: https://doi.org/10.1080/09650790802667402

Wenger, E. (2001). Comunidades de práctica: Aprendizaje, significado e identidad. Barcelona: Paidós.

Wenger, E., White, N. y Smith, J. D. (2009). Digital habitats:Sterwarding technology for communities. Portland: CPsquare. 